\title{
Bundesgesetz über genetische Untersuchungen beim Menschen (GUMG)
}

\section{Peter A. Suter}

Präsident «Kommission Medizinische Risikoprüfung» Schweizerischer Versicherungsverband SVV
Korrespondenz: Schweizerischer Versicherungsverband SVV C. F. Meyer-Strasse 14 CH-8002 Zürich Tel. 0442082880 Fax 0442082887

\section{Einleitung}

Bereits im Jahre 2004 hat das Parlament das neue Bundesgesetz über genetische Untersuchungen beim Menschen gutgeheissen. Die Inkraftsetzung durch den Bundesrat erfolgt nun auf den 1. April 2007. Für die Gesetzesartikel «Genetische Untersuchungen im Versicherungsbereich» gibt es keine Ausführungsbestimmungen. Die neuen gesetzlichen Regelungen haben aber Einfluss auf die praktische Durchführung der Antragsabwicklung in der Personenversicherung. Deshalb hat sich die «Kommission für Medizinische Risikoprüfung» des Schweizerischen Versicherungsverbandes (SVV) bereits im vergangenen Jahr den damit zusammenhängenden Fragen angenommen. Die dafür eingesetzte Arbeitsgruppe hat einerseits den Ärztlichen Untersuchungsbericht (Ausgabe 2006) an die neue Situation angepasst und andererseits Empfehlungen für die Mitgliedgesellschaften des SVV herausgegeben.

\section{Gesetzliche Regelungen}

An dieser Stelle seien kurz die für die Lebensversicherer relevanten gesetzlichen Artikel erwähnt. Es handelt sich um folgende Artikel:

- Artikel 3: Begriffe;

- Artikel 26: Untersuchungsverbot;

- Artikel 27: Nachforschungsverbot;

- Artikel 28: Zulässige Nachforschungen.

Speziell hervorzuheben ist, dass zwischen diagnostischen, präsymptomatischen und pränatalen Gentests zu unterscheiden ist. Für den behandelnden Arzt ist wichtig zu wissen, dass

- diagnostische Gentests bzw. Fragen zu Ergebnissen dazu von Gesetzes wegen nicht verboten sind;

- Nachforschungen bei präsymptomatischen Gentests nur in Ausnahmefällen und unter Einhaltung strikter Vorgehensweisen möglich sind und

- pränatale Gentests generell nicht verwertet werden dürfen.

Der genaue Wortlaut der vorerwähnten Artikel, aber auch das ganze GUMG, kann unter www. svv.ch (Fachpublikationen $\rightarrow$ Privatversicherungsrecht) eingesehen werden.

\section{Sicht der Lebensversicherer}

Aus Sicht der Lebensversicherer muss das neue Bundesgesetz durchaus kritisch betrachtet werden, begrenzt doch erstmals ein Gesetz im privaten Versicherungsbereich die Möglichkeiten der Risikoprüfung und nimmt eine Informationsasymmetrie in Kauf. Dadurch kann die Situation entstehen, dass die versicherte Person über risikorelevantes Wissen verfügt, das von Gesetzes wegen dem Lebensversicherer nicht zugänglich gemacht werden muss bzw. darf. Andererseits darf aber auch erwähnt werden, dass Resultate bereits durchgeführter Gentests für die Risikoprüfung in bestimmten Fällen verwendet werden dürfen und dass wir im internationalen Vergleich restriktivere - aber auch liberalere Regelungen finden.

\section{Neuer Ärztlicher Untersuchungsbericht der Schweizerischen Lebens- versicherungsgesellschaften}

Die Ausgabe 2006 des Ärztlichen Untersuchungsberichtes beinhaltet zwei wesentliche Änderungen sowie diverse kleinere Anpassungen, die ausschliesslich der qualitativen Verbesserung dienen.

\section{Informationspflicht}

Der Informationspflicht wird grosses Gewicht beigemessen. Aus diesem Grunde wird neu beim Ärztlichen Untersuchungsbericht einleitend darauf hingewiesen, was im Zusammenhang mit genetischen Untersuchungen und deren Ergebnissen erlaubt und was untersagt ist. Für den untersuchenden Arzt ist es wichtig zu wissen, dass keine Ergebnisse aus präsymptomatischen genetischen Untersuchungen erwähnt werden dürfen.

Auszug

Von Gesetzes wegen ist es den Versicherern untersagt, nach Ergebnissen von vorgeburtlichen genetischen Untersuchungen zu fragen. Fragen nach den Ergebnissen präsymptomatischer genetischer Untersuchungen (Untersuchungen zur Feststellung einer Krankheitsveranlagung, bevor Symptome aufgetreten sind) sind unter bestimmten Voraussetzungen zulässig. Sind diese Voraussetzungen erfüllt, erfolgt die Abklärung mit einem separaten Formular. Deshalb müssen solche Untersuchungsergebnisse im 
vorliegenden Fragebogen nicht angegeben werden. Freiwillig eingereichte Resultate dürfen von den Versicherern nicht verwendet werden.

Genetische Untersuchungen $\mathrm{zu}$ diagnostischen Zwecken, d.h. zur Abklärung bereits feststellbarer Krankheitssymptome, sind von der Gesetzesbestimmung nicht betroffen und müssen deklariert werden.

\section{Familienanamnese}

Die Frage zur Familienanamnese wurde der versicherungsmedizinischen Relevanz entsprechend modifiziert. Dies insbesondere auch darum, weil ja Fragen nach entsprechenden Tests in der Regel nicht zulässig sind. Zudem sind die Anforderungen des Datenschutzes zu berücksichtigen. Konkret wird neu nach entsprechenden Krankheiten nur noch vor dem Alter 55 gefragt. Nicht mehr anzugeben ist, bei welcher Person diese Krankheit aufgetreten ist, sondern neu ist zu erwähnen, in wie vielen Fällen die Krankheit vorkam.

Auszug

Sind bei Ihren Eltern, Geschwistern, Grosseltern vor Alter 55 neurologische Störungen, Herzkrankheiten, Schlaganfall, Diabetes mellitus, Krebs oder Erbkrankheiten vorgekommen?

\section{Übrige Anpassungen}

Als Beispiel sei hier erwähnt, dass neu der Bauchund der Hüftumfang angegeben werden müssen.

\section{Konkretes Vorgehen in der Praxis}

Sind alle gesetzlichen Voraussetzungen erfüllt und macht es aus Sicht der Risikoprüfung auch Sinn, kann in einem mehrstufigen und umständlichen Verfahren - so will es das Gesetz das Resultat einer präsymptomatischen genetischen Untersuchung erfragt werden.

Das Testergebnis darf aber nur verlangt werden, wenn es als zuverlässig gilt und für die Prämienberechnung relevant ist. Diesbezüglich wird im Einzelfall mit den spezialisierten Dien- sten der grossen Rückversicherungsgesellschaften der jeweils aktuelle Stand der Entwicklung abgeglichen. In einem ersten Schritt wird dann mittels Zusatzfragebogen die versicherte Person angefragt, ob eine genetische Untersuchung durchgeführt wurde. Allenfalls ist der zuständige Arzt zu nennen - Ergebnisse jedoch sind nicht mitzuteilen. Dieser Zusatzfragebogen muss dem beratenden Arzt der Gesellschaft geschickt werden, und dieser entscheidet dann, ob mit dem untersuchenden Arzt Kontakt aufzunehmen ist und das Ergebnis der präsymptomatischen genetischen Untersuchung verlangt werden soll. Dies natürlich nur, wenn alle Voraussetzungen gemäss GUMG erfüllt sind.

Detailliertere Informationen zum genauen Ablauf sind einerseits im GUMG (Art. 28) ersichtlich, und andererseits sei auf die Schriftenreihe Medinfo - Mitteilungen zu Themen der Lebensversicherung, Ausgabe 2006/1, zum Thema «Genetische Untersuchungen beim Menschen» verwiesen. Der Versand als Beilage der Schweizerischen Ärztezeitung Nr. 26 erfolgte im Juni 2006. Über www.svv.ch (Fachpublikationen $\rightarrow$ Medinfo) ist der Zugriff auf die elektronische Form möglich.

\section{Schlussbemerkungen}

In den nächsten Monaten werden die Lebensversicherer wohl noch nicht mit der doch recht komplexen Materie überhäuft. Mittel- und langfristig wird es aber schon so sein, dass das Thema seinen Platz einnehmen wird. Die Lebensversicherer werden mit Sicherheit alles unternehmen, um den vielfältigen Interessen gerecht zu werden, und durch die genaue Beobachtung des Marktes auch sicherstellen, dass einzelne Punkte (z.B. Summengrenzen) wieder thematisiert werden, wenn erste Erfahrungen gemacht werden konnten. 